\title{
Laser mode discrimination with intra-cavity spiral phase elements
}

\author{
Ram Oron ${ }^{\mathrm{a}, *}$, Yochay Danziger ${ }^{\mathrm{a}}$, Nir Davidson ${ }^{\mathrm{a}}$, Asher A. Friesem ${ }^{\mathrm{a}}$, \\ Erez Hasman ${ }^{\mathrm{b}}$ \\ ${ }^{\mathrm{a}}$ Weizmann Institute of Science, Department of Physics of Complex Systems, Rehovot 76100, Israel \\ ${ }^{\mathrm{b}}$ Technion - Israel Institute of Technology, Faculty of Mechanical Engineering, Haifa 32000, Israel
}

Received 11 May 1999; accepted 12 July 1999

\begin{abstract}
A novel method for discriminating and selecting a specific high order mode is presented. It is based on introducing spiral phase elements into a laser resonator, so as to improve the output beam quality over that when the laser operates with multi-modes, and yet maintain a relatively high output power. The theoretical analysis, along with experimental results with $\mathrm{CO}_{2}$ and Nd:YAG lasers are presented. The results reveal that a $50 \%$ increase of laser output power over that operating with $\mathrm{TEM}_{00}$ mode is possible. (C) 1999 Elsevier Science B.V. All rights reserved.
\end{abstract}

PACS: 42.60.Da; 42.60.Jf

\section{Introduction}

In a laser operating with many transverse modes the output power is relatively high but the output beam quality is inherently poor. To improve the beam quality, an aperture is typically introduced inside the resonator so as to reduce the number of modes. If the aperture is sufficiently small, only the fundamental $\mathrm{TEM}_{00}$ mode with a Gaussian shape will exist. Such a $\mathrm{TEM}_{00}$ mode results in the best beam quality. Typical criterion for the quality of the beam is $M^{2}$, which is smallest for the TEM $\mathrm{T}_{00}$ mode, with a value of 1 [1]. Unfortunately, this concomitantly leads to a significant reduction of the output power, because only a small volume of the gain medium is exploited.

\footnotetext{
* Corresponding author. E-mail: feoron@weizmann.weizmann.ac.il
}

Alternative approaches for improving the output beam quality and yet not significantly reducing the output powers involve the introduction of either diffractive elements [2] or conical laser mirrors [3] or other specially designed mirrors $[4,5]$ or discontinuous phase elements [6] into the laser resonator. These ensure that the laser will operate with only a single mode. Although the beam quality is improved, the diffractive elements and the mirrors approaches have radial dependence, so they are sensitive to axial displacement and thermal lensing.

Here we propose and demonstrate a new and more practical approach to obtain a laser operation with a single high order mode. This approach is based on azimuthal mode discrimination, by inserting into the laser resonator spiral phase elements (SPEs), that are essentially lossless for the desired high order mode, but introduce high losses to all other modes. Since these SPEs have no radial depen- 
dence, the effect of axial displacement and thermal lensing is reduced. Moreover, the resulting high order radial symmetric mode is stable and linearly polarized. In the following, we present the principle of our approach and the design of the SPEs, along with representative experimental results for $\mathrm{CO}_{2}$ and $\mathrm{Nd}$ :YAG lasers. These demonstrate that it is possible to operate the lasers with a single high order mode, so their output powers are higher than with the $\mathrm{TEM}_{00}$ mode and yet comparable beam quality.

\section{Basic principles}

We begin by considering the field distribution of a TEM mode inside a laser resonator. In cylindrical coordinates, the field distribution, denoted by $E(r, \theta)$ for a $\mathrm{TEM}_{\mathrm{pl}^{*}}$ (non-degenerate Laguerre-Gaussian) mode, can be expressed by [7]

$$
\begin{aligned}
E(r, \theta) & =E_{0} \varrho^{l / 2} L_{p}^{l}(\varrho) \exp (-\varrho / 2) \exp (-i l \theta) \\
& =R_{p}^{l}(r) \exp (-i l \theta),
\end{aligned}
$$

where $r$ and $\theta$ are the cylindrical coordinates, $E_{0}$ the magnitude of the field, $\varrho=2 r^{2} / w^{2}$ with $w$ as the spot size of the Gaussian beam, $L_{p}^{l}$ are the generalized Laguerre polynomials of order $p$ and index $l$, and $R_{p}^{l}(r)$ is an overall amplitude term that is a function of $r$. Since modes of opposite angular momentum have the same radial distribution $\left(R^{l}=\right.$
$R^{-l}$ ), they are usually degenerate, and appear simultaneously, leading to a $\mathrm{TEM}_{\mathrm{pl}}$ (degenerate Laguerre-Gaussian) mode with

$E(r, \theta)=R_{p}^{l}(r) \cos (l \theta)$.

Note, in general, the intensity distribution of the $\mathrm{TEM}_{\mathrm{pl}^{*}}$ mode will have a circularly symmetric annular shape, whereas that of the $\mathrm{TEM}_{\mathrm{pl}}$ will have lobes.

The degeneracy can be removed by discriminating and separating modes with opposite angular momentum. Specifically, by introducing spiral phase changes of the form $\exp (+N i \theta)$ or $\exp (-N i \theta)$ so that Eq. (2) reverts to Eq. (1) with $l=N$. In practice, the spiral phase change can be realized with SPEs. The SPEs in essence, change the phase of a wavefront passing through them, in accordance to either $\exp (+N i \theta)$ or $\exp (-N i \theta)$. A representative example of a SPE is shown in Fig. 1. Here for $N=1$, the discontinuity height is $\lambda$ for a reflective element and $\lambda /(n-1)$, where $n$ is the refractive index, for a transmittive element, both denoting a $2 \pi$ phase shift. As evident, a singularity appears in the origin of the SPE.

Two laser resonator configurations in which SPEs are incorporated are shown schematically in Fig. 2. Fig. 2(a) shows the basic laser resonator configuration with the two SPEs adjacent to the resonator

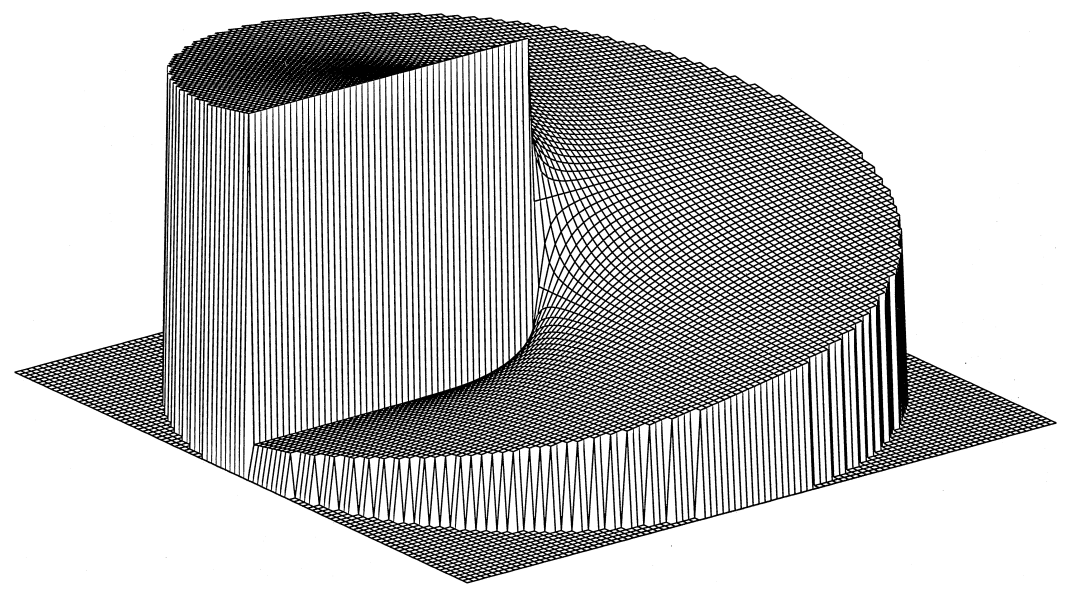

Fig. 1. Spiral phase element (SPE) with $N=1$. The discontinuity height represents a $2 \pi$ phase shift. 


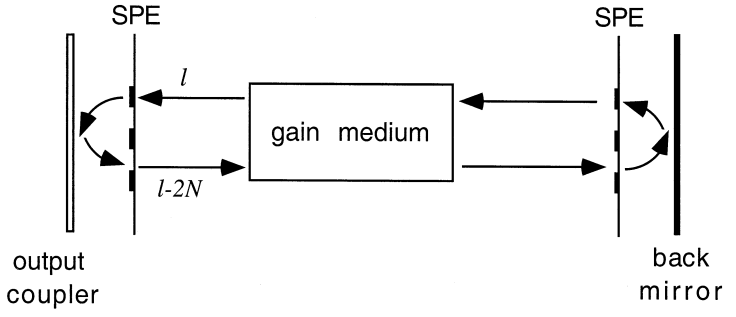

(a)

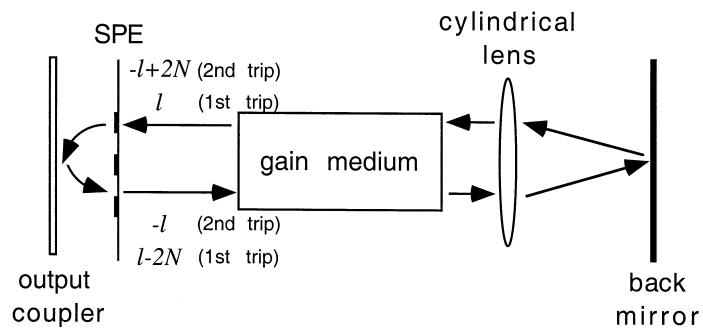

(b)

Fig. 2. Laser resonator configurations with SPEs (a) a configuration with two SPEs, each placed adjacent to a laser mirror; (b) a configuration with a single SPE, placed next to the output coupler, and a cylindrical lens (which reverses the angular phase) focused on the back mirror.

mirrors. The first SPE changes the angular node index of the mode passing twice through it by $-2 \mathrm{~N}$, i.e., mode of angular index $l$ changes into $l-2 N$ and the second SPE changes it back to $l$. With these SPEs the modes with an angular node index of $l=+N$ will be changed to those with $l=-N$. As a result, since $R^{l}=R^{-l}$, only these modes will maintain the same Laguerre-Gaussian radial distribution before and after passing through the SPEs. However, the radial distribution for the other modes will have the form of a combination of Laguerre-Gaussian modes, each with a different angular node index $l$, so they will be wider. Thus the losses for all modes having $l \neq N$ will be higher. Thus, in accordance to the design of the SPEs, it is possible to select a specific mode that will propagate inside the laser resonator. Moreover, it is possible to separate two $\mathrm{TEM}_{\mathrm{pl}^{*}}$ modes with opposite angular node indices, so as to lead to a pure $\mathrm{TEM}_{\mathrm{pl}^{*}}$ distribution. Specifically, the mode will be linearly polarized, with a field distribution of the form given by Eq. (1), i.e. doughnut shaped, rather than that of $\mathrm{TEM}_{\mathrm{pl}}$ of the form in Eq. (2), i.e. a distribution with distinct and separate lobes.

Fig. 2(b) shows a laser resonator configuration in which one of the SPEs is replaced by an element that reverses the angular phase (such as a cylindrical lens focused on the resonator mirror or a Porro prism). In this configuration, each passage through the SPE changes the angular node index of the mode passing through it by $-N$, and the angular phase reversing element, simply reverses the sign of the angular node index. Here again, the Laguerre-Gaussian radial distribution is maintained after each round-trip only for those modes having an angular node index of $l=N$. For other modes, however, the radial distribution is wider, resulting in significantly higher losses.

As evident from Fig. 2, the emerging output laser beam passes only once through the SPE. As a result the phase distribution of the output beam is constant. Thus, it converges to a relatively small single lobe in the far-field, with no need for any external element. This leads to a significant improvement in $M^{2}$, from $(1+2 p+l)$ for a degenerate $\mathrm{TEM}_{\mathrm{pl}}$ mode to $\sqrt{1+2 p+l}$ for the $\mathrm{TEM}_{\mathrm{pl}^{*}}$ mode. Finally we note that by interchanging the back mirror and the output coupler and adding an external cylindrical lens, it is possible to obtain the internal mode pattern outside the laser. Alternatively, this could be directly obtained by inserting a beam-splitter inside the resonator, so it will serve as an output coupler for getting the internal beam out of the resonator. In both cases, it is possible to further improve the beam quality with external phase elements [8], since the phase and amplitude of the output beam, emerging directly from the laser are well defined.

\section{Numerical analysis}

In order to evaluate the mode discrimination, we calculated the losses of the modes existing inside the laser resonator. For the calculation we represented the mirrors, SPEs, lensing, phase reversing element and propagation inside the resonator by matrix operators. Thus, the laser resonator can be characterized 
by the round-trip Fresnel propagation kernel $\kappa$, which is obtained as the product of the various matrix operators. By diagonalization of $\kappa$, we solve

$\kappa \cdot U_{\mathrm{m}}=\gamma_{\mathrm{m}} U_{\mathrm{m}}$,

where the resonator modes are the eigenvectors $U_{\mathrm{m}}$, and the round-trip losses of the modes are given by $1-\left|\gamma_{\mathrm{m}}\right|^{2}$, with $\gamma_{\mathrm{m}}$ being the eigenvalues of the round-trip Fresnel propagation kernel $\kappa$.

The results of our calculations are presented in Fig. 3. It shows the power loss per round trip as a function of the Fresnel number $N_{\mathrm{F}}$, for the different modes in the laser resonator. The Fresnel number is defined as $N_{\mathrm{F}}=a^{2} / \lambda L$, where $a$ is the aperture radius and $L$ is the resonator length. Fig. 3(a) shows the losses for a laser resonator configuration without any SPEs, whereas Fig. 3(b) shows the losses for a configuration with one SPE of $N=1$, and a cylindrical lens. As evident, the losses of the modes with $l=1$ are not affected by the SPE, while all other modes (including the fundamental $\mathrm{TEM}_{00}$ mode) suffer very high losses.

\section{Experimental procedure and results}

To experimentally verify our approach, we fabricated several SPEs that can be incorporated into a $\mathrm{CO}_{2}$ laser and a Nd:YAG laser. The SPEs for the $\mathrm{CO}_{2}$ laser were formed on GaAs substrates by means of a multi-level photo-lithographic process [9]. For our specific SPE we used 16 levels. For the experiments we used the laser resonator configuration shown in Fig. 2(b). The laser was a discharge pumped $\mathrm{CO}_{2}$ laser whose length was $60 \mathrm{~cm}$, the SPE was formed for $N=1$, and the cylindrical lens of $\mathrm{ZnSe}$ had a focal length of $7.5 \mathrm{~cm}$. The SPE and the cylindrical lens had AR coating for $\lambda=10.6 \mu \mathrm{m}$. In order to ensure that only the $\mathrm{TEM}_{01}$ * mode exists in the laser resonator, we inserted a single variable

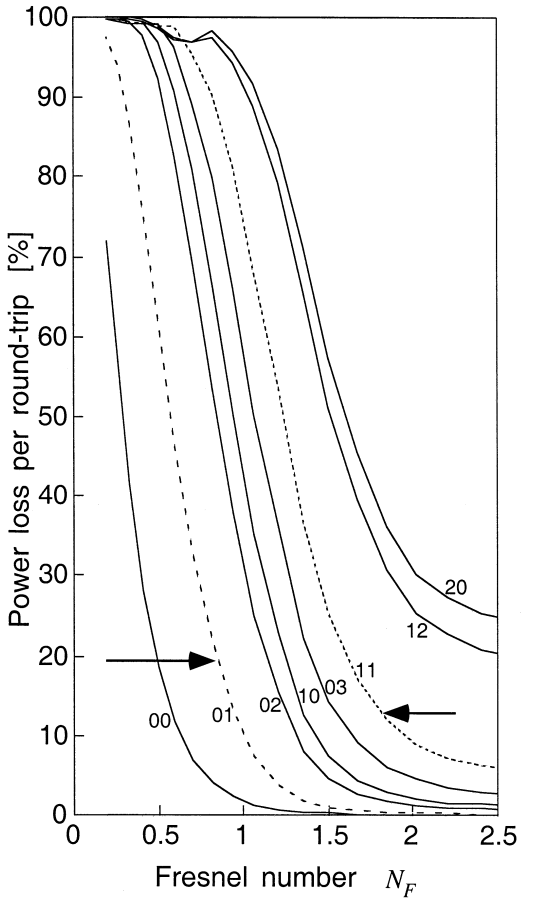

(a)

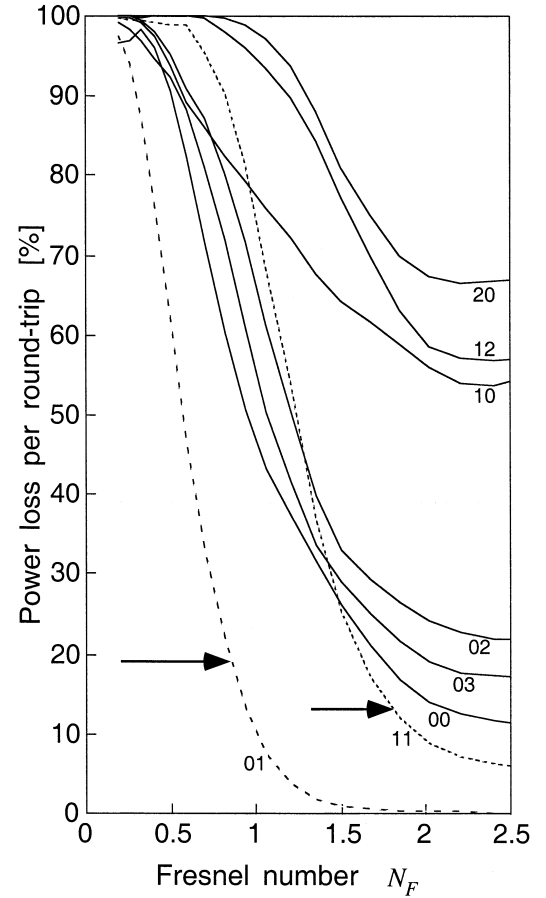

(b)

Fig. 3. Diffraction losses in a round-trip for various Laguerre-Gaussian transverse modes as a function of the Fresnel number $N_{\mathrm{F}}=a^{2} / \lambda L$. Modes with $l=1$ are indicated by arrows, dashed curve represents the $\mathrm{TEM}_{01 *}$ mode and dotted curve represents the TEM $11 *$ mode. (a) laser configuration with no SPE; (b) laser configuration with a single SPE of $N=1$, inserted into the resonator, as shown in Fig. 2(b). 
aperture which was adjusted until the emerging beam contained only one mode. This is equivalent to adjusting the Fresnel number, which in our experiments was 2 . We then detected the intensity distribution of the near-field and far-field by means of a pyro-electric camera.

The results are shown in Fig. 4 along with the corresponding calculated results from Eq. (1). Fig. 4(a) depicts the calculated near-field intensity distribution of the TEM $0_{01}$ mode. Fig. 4(b) and Fig. 4(c) present the corresponding experimental near field pattern and contour plot. The expected doughnut shape distribution is clearly evident in both calculated and experimental results. The non-uniformities in the experimental distribution arise from the 16 level discontinuities, caused by the $\pi / 8$ phase steps. Fig. 4(d) depicts the theoretical far-field intensity distribution, which is calculated by the Fourier transformation of the near-field pattern with a uniform phase. Fig. 4(e) and Fig. 4(f) depict the corresponding experimental far-field pattern and contour plot. The excellent agreement between the theoretical and the experimental patterns is clearly evident, indicating that all parts of the near field pattern are in phase. An $M^{2}$ value of 1.8 , and an output power of $3 \mathrm{~W}$ were obtained when the laser operated with the $\mathrm{TEM}_{01}$ * mode. The value of 1.8 for the $M^{2}$ is even better than the theoretical limit of $M^{2}=2$ for a

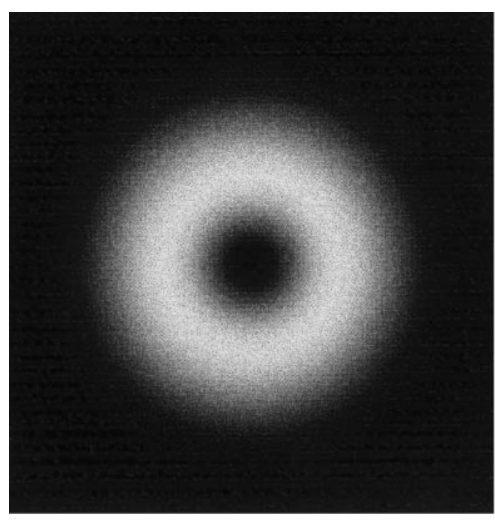

(a)

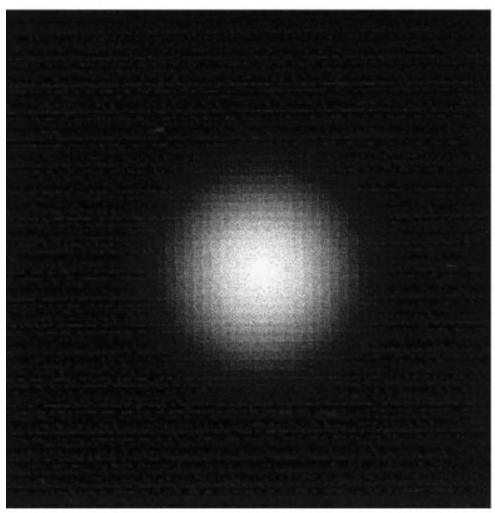

(d)

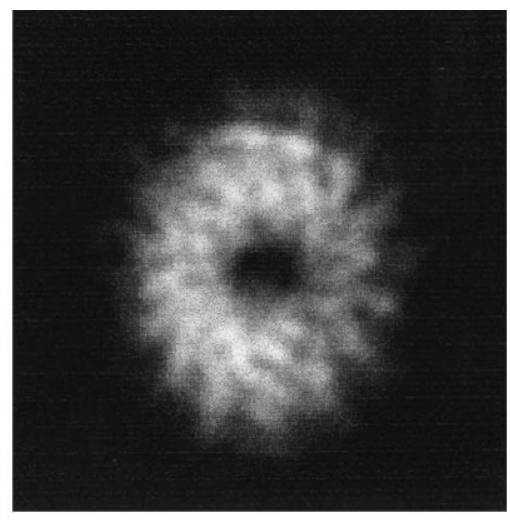

(b)

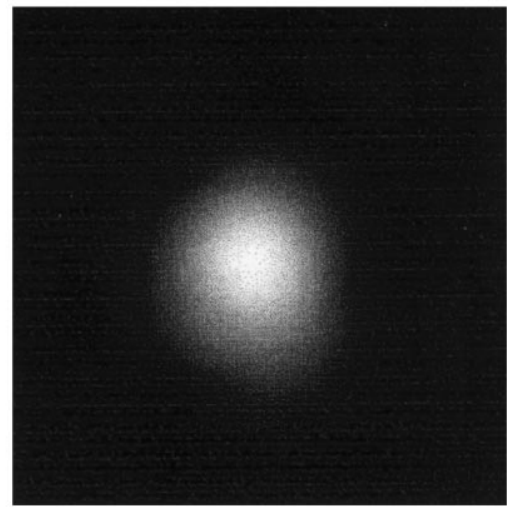

(e)

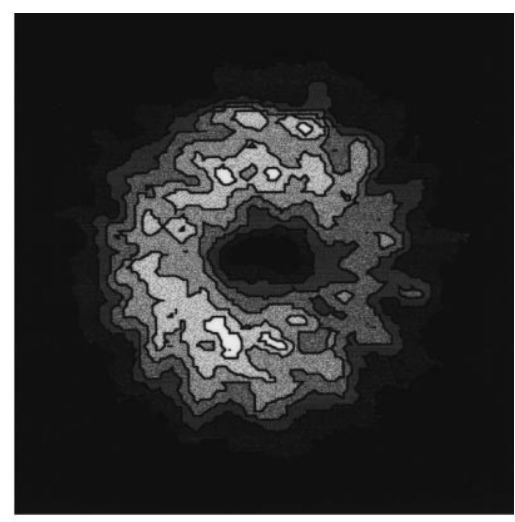

(c)

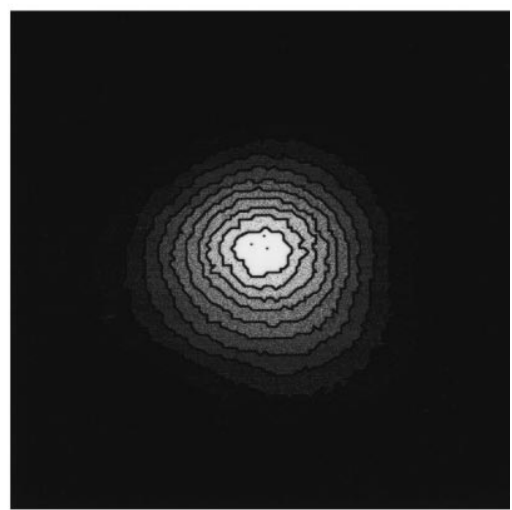

(f)

Fig. 4. Theoretical and experimental intensity distributions that emerge from a $\mathrm{CO}_{2}$ laser in which a SOE with $N=1$ was incorporated to obtain the $\mathrm{TEM}_{01}$ * mode. (a) theoretical near-field; (b) and (c) experimental near-field; (d) theoretical far-field; and (e) and (f) experimental far-field. 


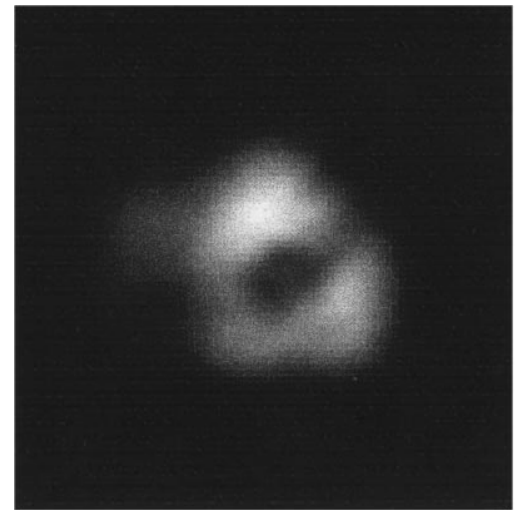

(a)

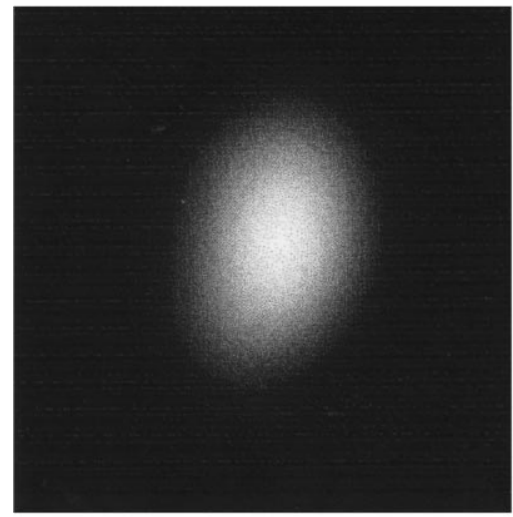

(c)

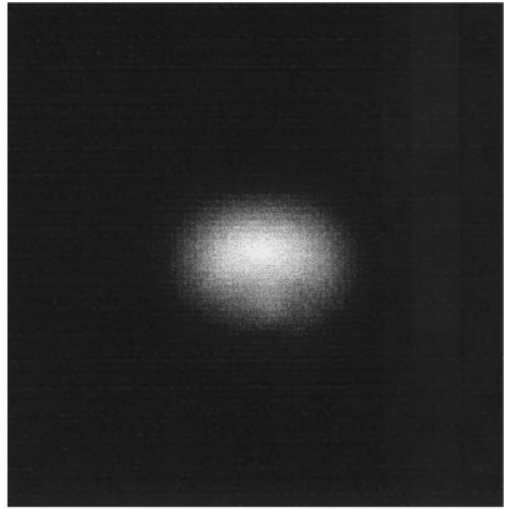

(b)

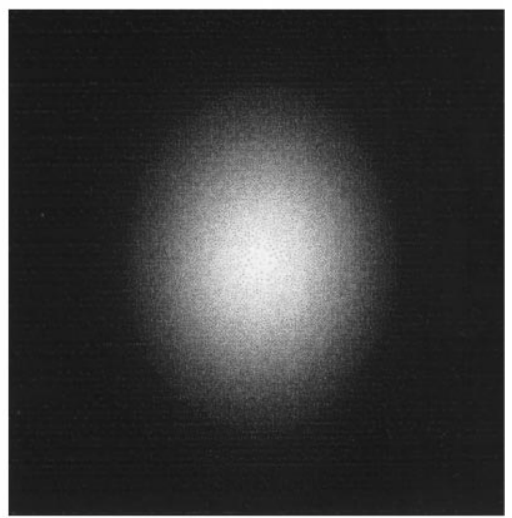

(d)

Fig. 5. Experimental intensity distributions that emerge from a Nd:YAG laser with SPEs to obtain the TEM $\mathrm{Tl}_{01}$ mode and simple experiment to obtain the fundamental $\mathrm{TEM}_{00}$ mode (a) $\mathrm{TEM}_{01} *$ near-field; (b) $\mathrm{TEM}_{00}$ near-field; (c) $\mathrm{TEM}_{01} *$ far-field; and (d) $\mathrm{TEM}_{00}$ far-field.

$\mathrm{TEM}_{01}$ mode. The power of $3 \mathrm{~W}$ is the same as that obtained by the same laser operating with the $\mathrm{TEM}_{00}$ mode despite the losses caused by the imperfections in the SPE ${ }^{1}$.

We also operated the laser with the single high order $\mathrm{TEM}_{02} *$ mode. This was done by exploiting a SPE with $N=2$. Here, the diameter of the near-field pattern was larger than the $\mathrm{TEM}_{01}$ * pattern, whereas the far-field pattern still converged to a narrow single lobe. This indicates that the phase distribution of the near-field pattern was uniform.

\footnotetext{
${ }^{1}$ To measure the losses due to imperfections in fabrication and AR coating of the SPE, we introduced a uniform phase section of the SPE into a laser operating with $\mathrm{TEM}_{00}$ mode. The output power decreased to $2.3 \mathrm{~W}$.
}

The SPEs that were incorporated into the Nd:YAG laser were formed on fused silica substrates. For these experiments we used the laser resonator configuration, with two SPEs, as shown in Fig. 2(a). The laser was a flashlamp pumped Nd:YAG laser whose length was $60 \mathrm{~cm}$, and both SPEs were formed for $N=1$, and AR coated for $\lambda=1.06 \mu \mathrm{m}$. Here again, we applied an internal aperture in order to achieve a single mode operation. We then detected the intensity distributions of the near-field and far-field by means of a CCD camera.

The experimental results with the Nd:YAG laser are shown in Fig. 5. Fig. 5(a) presents the near field pattern of the $\mathrm{TEM}_{01} *$ mode, with the expected doughnut shape distribution. Fig. 5(b) depicts the corresponding fundamental $\mathrm{TEM}_{00}$ mode pattern 
(with no SPEs). Fig. 5(c) depicts the TEM T1* $^{*}$ mode far-field pattern, where the single main lobe is evident, indicating again, that all parts of the near-field pattern are in phase. Finally, Fig. 5(d) depicts the fundamental $\mathrm{TEM}_{00}$ mode far-field pattern, whose cross section area is, as expected, similar to that of the higher order $\mathrm{TEM}_{01}$ * mode. As evident, there is some asymmetry in the intensity distributions. We attribute this asymmetrical behavior to gain non-uniformities. An output power of 5.2W was obtained when the laser operated with the $\mathrm{TEM}_{01}{ }^{*}$ mode, which was higher by up to 50 percent with respect to that obtained by the same laser operating with the $\mathrm{TEM}_{00}$ mode and no SPEs.

\section{Concluding remarks}

We have shown that it is possible to discriminate and select a specific high order mode by inserting spiral phase elements into the laser resonator. Moreover, it is possible to discriminate between modes of opposite angular node indices, and to improve the $M^{2}$ value. These elements are lossless, and insensitive to longitudinal displacement or thermal lensing, and can be successfully incorporated into variety of lasers. The selected high order radial symmetric mode converge into a single lobe in the far-field, as for the $\mathrm{TEM}_{00}$ mode. Finally, the output powers with a single higher order mode is higher than that with the fundamental $\mathrm{TEM}_{00}$ mode.

\section{Acknowledgements}

This research was supported by Pamot Venture Capital Fund.

\section{References}

[1] A.E. Siegman, Proc. SPIE 1224 (1990) 2.

[2] J.R. Leger, D. Chen, K. Dai, Opt. Lett. 19 (1994) 1976.

[3] M. Rioux, P.A. Belanger, M. Cormier, Appl. Opt. 16 (1977) 1791.

[4] M. Piche, D. Cantin, Opt. Lett. 16 (1991) 1135.

[5] P.A. Belanger, R.L. Lachance, C. Pare, Opt. Lett. 17 (1992) 739.

[6] R. Oron, Y. Danziger, N. Davidson, A.A. Friesem, E. Hasman, Appl. Phys. Lett. 74 (1999) 1373.

[7] W. Koechner, Solid State Laser Engineering, Springer-Verlag, Berlin, 4th ed., 1996.

[8] N. Davidson, A.A. Friesem, E. Hasman, Appl. Phys. Lett. 61 (1992) 381.

[9] E. Hasman, N. Davidson, A.A. Friesem, Opt. Lett. 16 (1991) 423. 